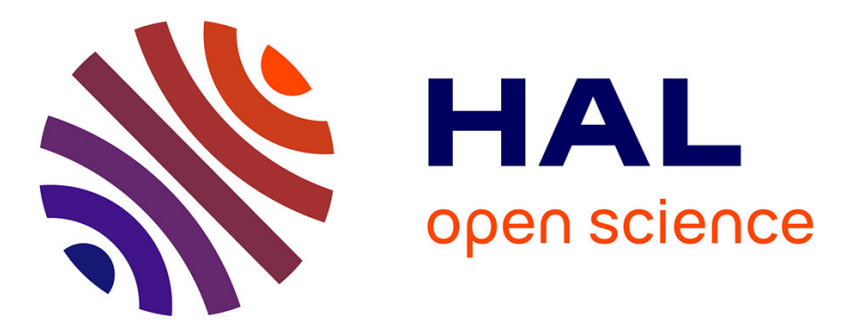

\title{
Sensori-motor strategic variations and sequential effects in young and older adults performing a Fitts' task
}

\author{
Céline Poletti, Rita Sleimen-Malkoun, Patrick Lemaire, Jean-Jacques
}

Temprado

\section{- To cite this version:}

Céline Poletti, Rita Sleimen-Malkoun, Patrick Lemaire, Jean-Jacques Temprado. Sensori-motor strategic variations and sequential effects in young and older adults performing a Fitts' task. Acta Psychologica, 2016, 163, pp.1 - 9. 10.1016/j.actpsy.2015.10.003 . hal-01460213

\section{HAL Id: hal-01460213 \\ https://hal-amu.archives-ouvertes.fr/hal-01460213}

Submitted on 17 Feb 2017

HAL is a multi-disciplinary open access archive for the deposit and dissemination of scientific research documents, whether they are published or not. The documents may come from teaching and research institutions in France or abroad, or from public or private research centers.
L'archive ouverte pluridisciplinaire HAL, est destinée au dépôt et à la diffusion de documents scientifiques de niveau recherche, publiés ou non, émanant des établissements d'enseignement et de recherche français ou étrangers, des laboratoires publics ou privés. 


\title{
Sensori-motor strategic variations and sequential effects in young and older adults performing a Fitts' task is $^{2}$
}

\author{
Céline Poletti ${ }^{\mathrm{a}, \mathrm{b}}$, Rita Sleimen-Malkoun ${ }^{\mathrm{b}, \mathrm{c}}$, Patrick Lemaire ${ }^{\mathrm{a}}$, Jean-Jacques Temprado ${ }^{\mathrm{b}, *}$ \\ a Aix-Marseille Université, CNRS, UMR 7290, E IUF, 13331 Marseille Cedex 03, France \\ b Aix-Marseille Université, CNRS, ISM UMR 7287, 13288 Marseille Cedex 09, France \\ c Aix-Marseille Université, Inserm, INS UMR 1106, 13385 Marseille Cedex 05, France
}

\section{A R T I C L E I N F O}

\section{Article history:}

Received 13 May 2015

Received in revised form 23 October 2015

Accepted 26 October 2015

Available online $\mathrm{xxxx}$

\section{Keywords:}

Aging

Fitts' task

Kinematic strategies

Sequential effects

\begin{abstract}
A B S T R A C T
The present study aimed at investigating age-related changes in strategic variations and sequential effects in discrete Fitts' aiming task. Three sequential effects were investigated, namely trial sequential difficulty effects (TSDE), strategy sequential difficulty effects (SSDE), and strategy repetition effects (SRE). After generalizing previously observed aging effects on strategic variations, our results showed that movement times were longer when performed after harder ID level than when following easier ID level (TSDE). We also observed SSDE, such that is movement times were longer when participants executed a strategy of intermediate difficulty (i.e., the progressive-deceleration strategy) after having used a more difficult strategy (i.e., the undershoot strategy) on the previous trial than after an easier strategy (i.e., the one-shot strategy). These sequential difficulty effects related to both difficulty and strategy were similar in young and older adults. In addition, we found that across two successive trials, participants tended to repeat the one-shot strategy the most often and the undershoot strategy the least often, with repetition rates of the progressive-deceleration strategy being in-between (SRE). Finally, age-related differences in strategy repetition effects varied with strategies (e.g., they were largest for the one-shot strategy). These findings have important implications for deciphering processes responsible for sequential effects in sensori-motor tasks as well as in cognitive tasks in general, and for our understanding of processes underlying sensori-motor performance in young and older adults.
\end{abstract}

C 2015 Elsevier B.V. All rights reserved.

\section{Introduction}

Aging is characterized by a decrease of cognitive and motor performance. Thus, an important issue is to investigate the underlying mechanisms of these age-related changes. In the motor domain, Fitts' task is a robust paradigm that has been extensively used to study the contribution of cognitive and sensori-motor processes to the control of rapid and accurate movements, in both young and older adults (Ketcham, Seidler, Van Gemmert, \& Stelmach, 2002; Pratt, Chasteen, \& Abrams, 1994; Rey-Robert, Temprado, Lemaire, \& Berton, 2012, Temprado et al., 2013). Fitts' task consists of performing rapid-aiming movements from a starting position toward a target, leading participants to adopt an optimal compromise between speed and accuracy. The width (W) and/ or distance (D) of the target can be varied to modulate task difficulty (Fitts, 1954; Fitts \& Peterson, 1964). In the framework of information theory, the index of difficulty (ID $=\log _{2}(2 \times \mathrm{D} / \mathrm{W})$ measures the

\footnotetext{
it This research was supported by grants from the Agence Nationale de la Recherche (Grant \# ANR-13-BSH2-0005-01 and Grant \# ANR-2010-BLAN-1912-01).

* Corresponding author at: Institut des Sciences du Mouvement, Aix-Marseille Université, Faculté des Sciences du Sport, 163 Route de Luminy, BP 910, 13288 Marseille, Cedex, France.

E-mail address: jean-jacques.temprado@univ-amu.fr (J.-J. Temprado).
}

amount of information that is required to ensure an optimal speedaccuracy trade-off. Accordingly, movement time (MT) is related to D and $\mathrm{W}$ according to the following relation: $\mathrm{MT}=\mathrm{a}+\mathrm{b} \times \mathrm{ID}$, with $\mathrm{a}$ and $b$ empirically determined constants. This linear relation - so called Fitts' law - quantifies the efficiency function of the informationprocessing system in central nervous system: The steeper the slope, the longer it takes to process a fixed amount of information.

Fitts' task is prone to differences in movement times and efficiency functions, which may arise from a coalition of external (e.g., the level of task difficulty) and internal (e.g., aging) constraints. For instance, several studies showed that increasing task difficulty (ID) led to larger slowing of movement execution in older than in young participants (Haaland, Harrington, \& Grice, 1993; Ketcham et al., 2002; Rey-Robert et al., 2012; Sleimen-Malkoun, Temprado, \& Berton, 2013; Teeken et al., 1996; Temprado et al., 2013; York \& Biederman, 1990; Welford, Norris, \& Shock, 1969). This observation was considered as a signature of the general slowing of information processing speed with aging (Rey-Robert et al., 2012; Sleimen-Malkoun et al., 2013; Temprado et al., 2013), which is also one of the hallmarks of age effects on the cognitive system (e.g., Bashore, Ridderinkhof, \& van der Molen, 1997; Birren, Woods, \& Williams, 1980; Cerella, 1985; Salthouse, 1996).

In a recent paper (Poletti, Sleimen-malkoun, Temprado, \& Lemaire, 2015), we used Fitts' paradigm to explore a complimentary facet of 
age-related changes that cannot be assessed through chronometric analyses alone that is, strategic variations underlying sensori-motor behavior. Specifically, by studying the different kinematic profiles, we empirically documented the usefulness of the strategy perspective for the understanding of strategic control and aging effects in the sensorimotor domain.

The identification of kinematic profiles was based on the current distinction between an initial impulse phase (i.e., a ballistic primary submovement characterized by a rapid movement that brings the limb near the target) and an online control phase (a secondary corrective submovement during which the target is approached) (Woodworth, 1899; Meyer, Abrams, Kornblum, Wright, \& Smith, 1988; see Elliott, Helsen, \& Chua, 2001, Elliott et al., 2010, for reviews). Based on this two-component model, we capitalized on Dounskaia, Wisleder, and Johnson's (2005) study, in which a further distinction was introduced according to the presence of three different secondary submovement types (i.e., Types 1, 2, and 3) during the secondary movement phase. According to Dounskaia et al. (2005), each of these different submovements has a specific kinematic signature: Type 1 secondary submovements are used to correct for overshooting; Type 2 submovements are used to correct for undershooting; and Type 3 submovements refer to finest corrections involved in the progressive deceleration to stop within the target. In Fradet, Lee, and Dounskaia's (2008) study, it has been shown that older adults more often used movement kinematics with corrective secondary submovements. This result is consistent with recent papers by Van Halewyck et al. (2014) (see also Van Halewyck et al., 2015) who showed that age-related differences in movement kinematics were prominently observed in secondary submovements.

In our previous study, the different kinematic profiles were considered as separate strategies in reference to the theoretical framework proposed by Lemaire and Siegler (1995; see also Lemaire, 2010) in the cognitive domain. A strategy can be defined as "a procedure or a set of procedures used to achieve a higher level goal" (Lemaire \& Reder, 1999, p. 365). Accordingly, depending on the type of submovement observed in the kinematic profile (i.e., no submovements, Type 1, 2, and 3 submovements), specific strategies were identified that is, the one-shot, overshoot, undershoot and progressive-deceleration strategies, respectively. For the one-shot strategy, participants correctly estimated the distance of the target and did not have to make a corrective submovement to reach the target. For the overshoot strategy, participants overestimated the distance and had to make a secondary corrective sub-movement by reversing direction to reach the target. For the undershoot strategy, participants underestimated the distance and had to make a secondary corrective sub-movement (reacceleration) to reach the target. For the progressive deceleration strategy, participants performed a movement that brought their arm near the target and then slowly approached the target, leading to a lengthening of the deceleration phase. Accordingly, our results showed that these distinct kinematic patterns fulfill the currently accepted criteria to empirically distinguish among strategies, namely: (a) main effects of strategies on collected measures (strategy use and strategy performance), (b) interaction effects between the strategy factor and one or several factors on strategy use and strategy performance, and (c) age-related differences in strategy use and performance. More specifically, Poletti et al. (2015) found that young and older participants used the four identified strategies, but differed in strategy distributions (i.e., how often young and older adults use each available strategy), strategy execution (i.e., how quick they are with each available strategy), and strategy selection (i.e., how they choose among available strategies on each trial). Specifically, in this study, it was found that: (a) young adults used significantly more strategies than older adults, (b) compared to young adults who tended to use the one-shot strategy, older adults preferred the two strategies involving submovements (i.e., the undershoot and progressive-deceleration strategies), and (c) young adults were faster than older adults whatever the strategies they used. These differences were also modulated by trial difficulty. Indeed, age-related differences in strategy performance were larger for the most difficult trials. These results showed that age-related changes of cognitive and sensorimotor processes involved in manual aiming movements had consequences on the kinematic strategies used to perform the task. In addition, the direction of these changes was roughly similar to those observed in cognitive (see Lemaire, 2015 for an overview). For example, in numerical cognition, older participants have been found to: a) have a smaller strategy repertoire than young participants (Hodzik \& Lemaire, 2011; Lemaire \& Arnaud, 2008), b) tend to use easier strategies even if those strategies do not yield the best performance (Gandini, Lemaire, \& Dufau, 2008), c) select the best strategy on fewer problems (Lemaire, Arnaud, \& Lecacheur, 2004), and d) are slower when using harder strategies (Gandini et al., 2008). Thus, these observations can be considered as empirical evidence of the existence of four different strategies instead of different variants of the same strategy, which would be not influenced by different factors (see Dunlosky, Hertzog, \& Powell-Moman, 2005, for supporting evidence). All in all, these results have encouraged us to investigate sequential effects, which are another prominent phenomenon of strategic variations in cognitive tasks. In our previous study, inter-trial variability in strategy use was observed, despite the fact that both young and older adults had to perform 12 consecutive trials for each ID conditions. The origins of this variability is unknown but the question arises as to whether movement times observed on a current trial, for a given level of task difficulty, could be influenced by the difficulty level and/or by the strategy used on the previous trial. For these reasons, in the present study, we switched between easy and difficult trials to raise the variability of the task within the same block.

In the cognitive tasks, these effects are currently referred to as problem sequential difficulty effects (PSDE) or strategy sequential difficulty effects (SSDE), respectively (Schneider \& Anderson, 2010; Uittenhove \& Lemaire, 2012, 2013a, 2013b; Uittenhove, Poletti, Dufau, \& Lemaire, 2013). For instance, as an illustration of the PSDE, Schneider and Anderson (2010) found that solving arithmetic problems was slowed after difficult problems relative to after easy problems. Furthermore, Uittenhove and Lemaire (2012) reported SSDE, such that a strategy was executed more slowly if it followed a harder strategy than if it followed an easier strategy. In addition, Lemaire and Leclère (2014a, 2014b) found that participants tended to repeat the same strategy over two successive trials, a phenomenon they called "strategy repetition effects" (SRE).

Following Schneider and Anderson (2010); Uittenhove and Lemaire (2012) explained these sequential effects by assuming lesser availability of executive resources after execution of a difficult trial/strategy. They hypothesized that difficult strategies or difficult problems temporarily consume central cognitive resources such as executive functions and/ or working memory. Consequently, the temporary depletion of executive resources results in slowing down execution of the next strategy and/or solving the next problem. The present study is grounded on this framework.

\subsection{Objectives and hypotheses}

It has been previously suggested that executive functions are strongly involved in Fitts' task, especially during the deceleration phase (see Rey-Robert et al., 2012; Sleimen-Malkoun et al., 2013; Temprado et al., 2013). Accordingly, one can expect to observe sequential effects even in this type of task. The general objective of the present study was to test this general hypothesis. If confirmed, such findings would update the factors and mechanisms that determine participants' performance, which have never been yet investigated in the motor control literature constitute. In addition, it would be a first step to generalize agerelated differences in strategic variations to the motor domain, thereby suggesting that executive functions influence strategic variations whatever the (mental or motor) tasks. 
To assess sequential effects in Fitts' task, we manipulated the sequences of difficulty levels by manipulating the size of the target. In doing that, we aimed at determining whether participants' performance on a given trial was influenced by the difficulty level of the preceding trial (TSDE) and/or of the strategy used on the preceding trial (SSDE). We also aimed at determining whether participants tend to repeat the same strategy on successive trials. Finally, we tested whether these sequential effects increase with aging, presumably as the result of the depletion of information processing speed and executive functions.

Primarily, we analyzed strategic variations and compared them to those reported in Poletti et al. (2015) above and beyond differences in designs between the two studies. Then, a series of analyses investigated sequential effects. First, we tested the so-called trial sequential difficulty effects (TSDE). In these effects longer movement times on trials were expected after harder ID level relative to after lower ID level. Second we tested the so-called strategy sequential difficulty effects (SSDE). Following Poletti et al.'s (2015) findings, we classified the different strategies as a function of their presumed difficulty level, with the harder strategy being the one requiring longer movement times. Thus, the one-shot strategy was considered the easier, the undershoot strategy the most difficult, and the progressive-deceleration strategy of intermediate difficulty. Accordingly, we expected that MTs should be longer with the strategy of intermediate difficulty (i.e., the progressivedeceleration strategy) after executing the most difficult strategy (i.e., the undershoot strategy) on the immediately preceding trial than after the easier strategy (i.e., the one-shot strategy). We expected both of these (TSDE, SSDE) sequential effects to be larger in older than in young adults, as a result of age-related decrease in information processing resources and executive functions efficiency. Third, we tested the socalled strategy repetition effects (SRE). Following previous findings by Lemaire and Leclère (2014a, 2014b) in cognitive tasks, we predicted that older participants would repeat more frequently the same strategy than young adults. Thus, we expected to observe a larger SRE in older than in young participants.

\section{Method}

\subsection{Participants}

Twenty-four young adults ( 14 women; mean age $=27.2$ years) and 24 older adults ( 11 women; mean age $=69.6$ years) were tested. Young adults were undergraduates from Aix-Marseille University (Marseille, France), who voluntarily participated in this experiment. Older adults were volunteers from the community of Marseille, with no cognitive or health-related problems. All older adults had scores larger than 27 $(\mathrm{M}=29.5)$ in the Mini Mental-State Examination (MMSE; Folstein, Folstein, \& McHugh, 1975); therefore none were excluded. After a presentation of the experiment, we collected information about each participant's sex, age, and the number of years of formal education (see Table 1 for participants' characteristics). Then, each participant signed an informed written consent, approved by the local ethic committee of Aix-Marseille University, in accordance with the ethical standards laid down in the Declaration of Helsinki.

Table 1

Participants' characteristics.

\begin{tabular}{|c|c|c|}
\hline Variable & Younger adults & Older adults \\
\hline $\mathrm{N}$ & 24 & 24 \\
\hline Age (SD) & $27.2(2.6)$ & $69.6(6.6)$ \\
\hline Years of education $(\mathrm{SD})^{*}$ & $16.7(2.3)$ & $13.8(2.5)$ \\
\hline MMSE (SD) & - & $29.5(0.7)$ \\
\hline
\end{tabular}

Note. MMSE $=$ Mini-mental state examination.

$* \mathrm{t}(46)=4.21 ; \mathrm{p}<.001$.

\subsection{Procedure}

Participants were comfortably seated in front of a Wacom graphic tablet (Intuos4 XL) positioned on the tabletop and connected (via a USB port) to a portable PC. They had to perform pointing movements with the right arm, by sliding a hand-held non-marking stylus (Wacom, Generation 2 tip sensor) over the surface of the tablet, from a starting position toward a target position. Sliding movements were performed in the anterior-posterior direction, with a combination of shoulder flexion and elbow extension. The trunk position was restricted by the chair-back position and the front edge of the table (see Fig. 1). Displacements of the stylus on the tablet were recorded using a customized software (ICE) developed at the laboratory (Institute of Movement Sciences, Marseille) at a sampling frequency of $250 \mathrm{~Hz}$.

Before each trial, participants were asked to hold still the stylus on the starting position until they heard a beep. Then, they were instructed to keep optimal speed-accuracy trade-off that is "to move as fast as possible from the starting position to the target and to stop on it". Before the experimental task, participants were allowed to complete two (unrecorded) familiarization trials for both ID conditions. Then, participants completed the Fitts' task in 12 blocks of 16 trials each, for a total of 192 targets to reach, with a break in-between each block. Within the same block, half the trials were preceded by a trial of similar difficulty and half by a trial of different difficulty. Moreover, the order of presentation of easier and harder trials was counterbalanced within each block.

\subsection{Task conditions}

The starting position of Fitts' task, represented as a black circle of $0.2 \mathrm{~cm}$ diameter, was always aligned with the center of the target, represented by a red horizontal rectangle $(10 \mathrm{~cm} \times$ various width values). Distance from starting position was held constant $(26 \mathrm{~cm})$, and target widths were either $6.5 \mathrm{~cm}$ or $0.4 \mathrm{~cm}$, yielding two ID levels of 3 and 7 bits, respectively. Each participant performed 192 trials, of which half were easy (ID level of 3 bits) and half were hard (ID level of 7 bits). Moreover, half the easy trials were placed on the right side of the

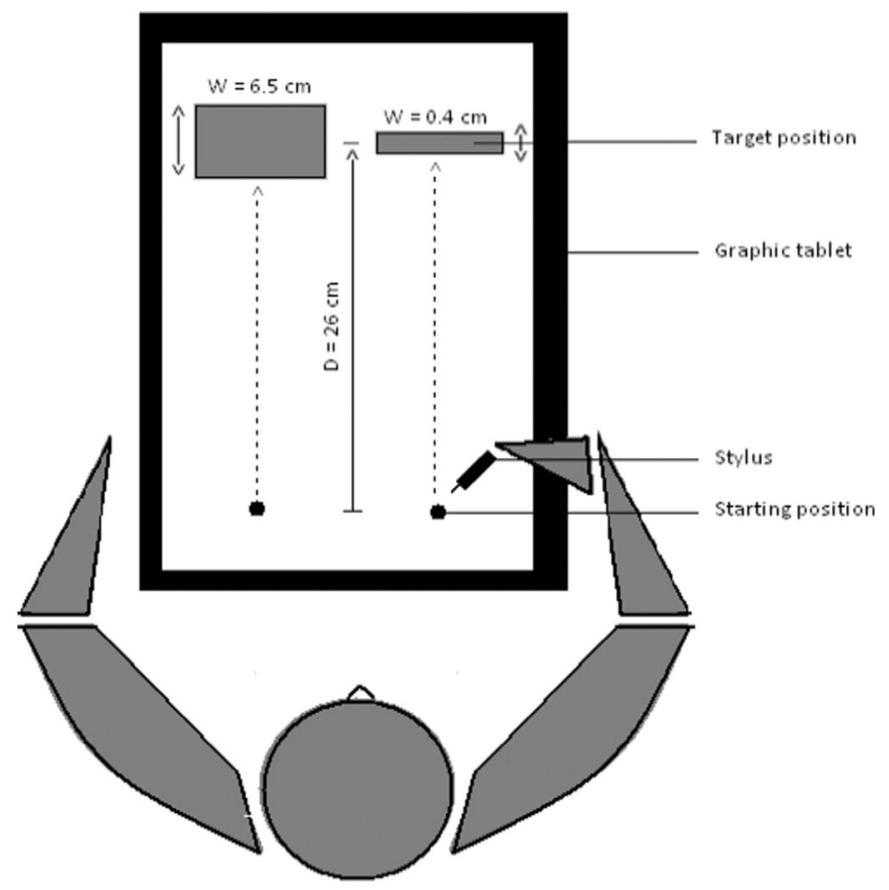

Fig. 1. Illustration of the Fitts' task with the two different ID conditions ( 3 and 7 bits) obtained via manipulation of target width (i.e., $6.5 \mathrm{~cm}$ and $0.4 \mathrm{~cm}$ ) under a target-distance of $26 \mathrm{~cm}$. 
sheet, and half on the left side (the same for the hard trials). In order to test sequential difficulty effects, half the trials were preceded by a trial of similar difficulty and half by a trial of different difficulty.

\subsection{Variables and data processing}

The pen-tip raw displacement data were filtered using a secondorder dual pass (no phase-lag) Butterworth filter with a cut-off frequency of $10 \mathrm{~Hz}$. First, second, and third derivatives of displacement (velocity, acceleration, and jerk, respectively) were then computed in MATLAB (MathWorks, v.7.5.0 R2007b). Movement onset and offset were determined on the basis of velocity profiles using the optimal algorithm of Teasdale, Bard, Fleury, Young, and Proteau (1993). The critical velocity threshold was obtained by multiplying peak velocity by 0.05 .

First, this procedure allowed us to calculate for each trial, in each condition, the movement time (MT) corresponding to the time to reach the target and the effective target width (We). The effective target width (We) was calculated from the standard deviation of movement end points (Mackenzie, 1992) using the following formula: We = $2 * 1.96 * \mathrm{SD}_{\mathrm{A}}$; where $\mathrm{SD}_{\mathrm{A}}$ is the standard deviation of movement amplitude, and 1.96 is the boundary of a normal distribution at $95 \%$. Then, to check whether the prescribed IDs were respected or not (SleimenMalkoun, Temprado, Huys, Jirsa, \& Berton, 2012), we compared the distributions of movement end points (centered on mean movement amplitude and bounded by calculated We), and the prescribed ones (centered on target distance and bounded by target edges). These comparisons yielded no significant statistical differences ( $\mathrm{ts}<1$ ).

Second, following a similar procedure as those previously used by Poletti et al. (2015), these data were distinguished into the primary and secondary submovements. The primary submovement was characterized by a rapid movement that brings the limb near the target and the optional secondary submovement corresponded to an online control phase during which the target is approached (Woodworth, 1899). Then, following Meyer et al. (1988), the end of the primary submovement was defined as the moment of time when one of the following three events occurred after the velocity reached its peak: (a) the velocity crossed zero, changing from positive to negative, (b) the acceleration crossed zero, changing from negative to positive, or (c) the jerk crossed zero, changing from positive to negative. This procedure allowed us to calculate kinematic variables that were related to the type of the secondary submovement identified (see Dounskaia et al., 2005). Each secondary submovement has been used to distinguish types of strategies. As can been seen in Fig. 2, four available strategies that participants used to reach the target were identified: (a) the strategy based on velocity zero-crossing constituted the overshoot strategy (i.e., the participant overestimated the distance and had to make a secondary corrective submovement by reversing direction to reach the target); (b) the strategy based on acceleration zero-crossing reflected the
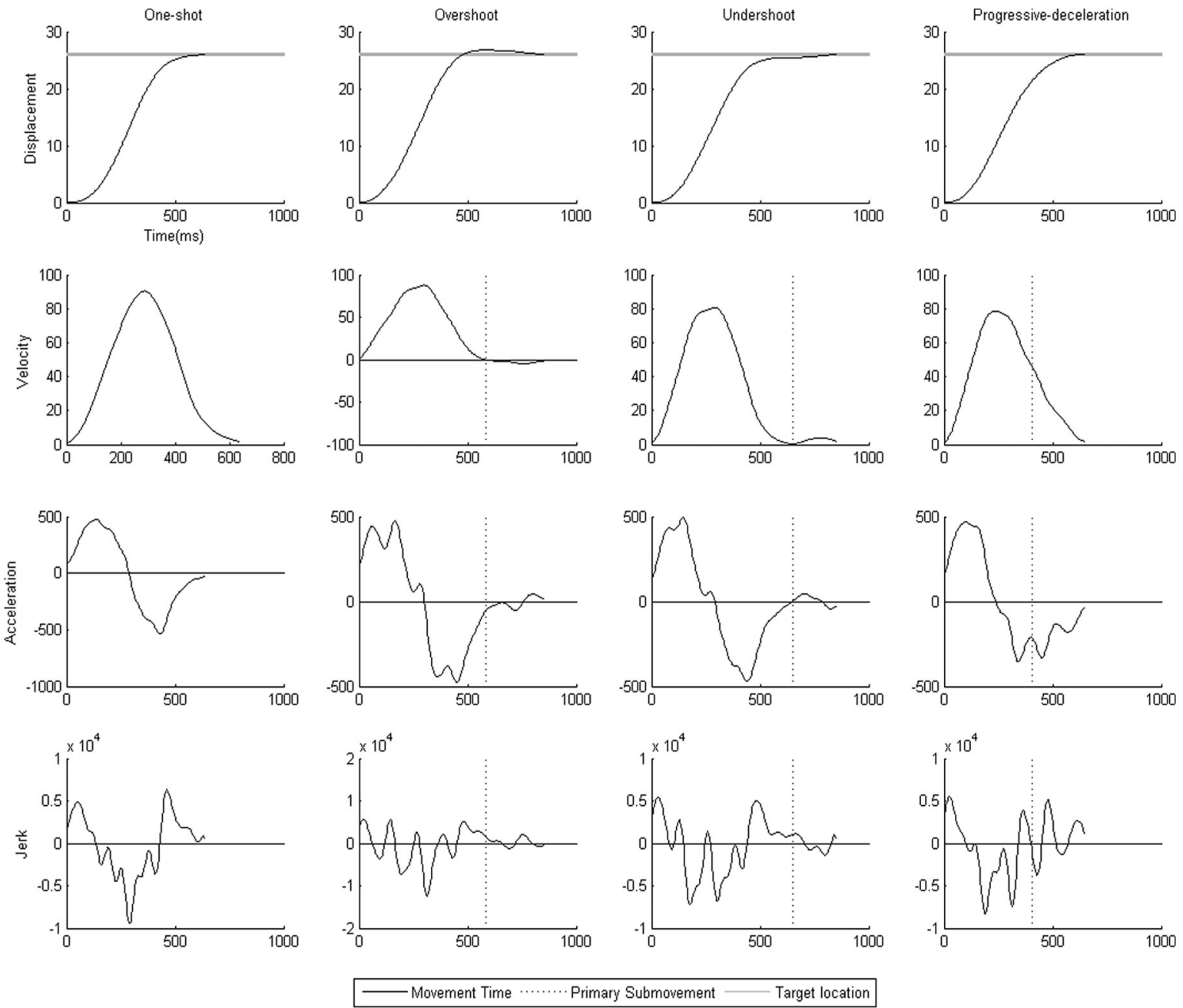

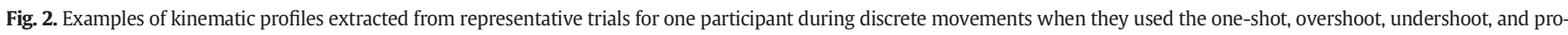

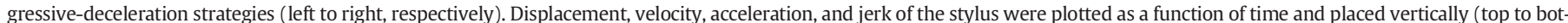

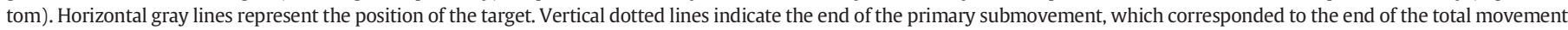

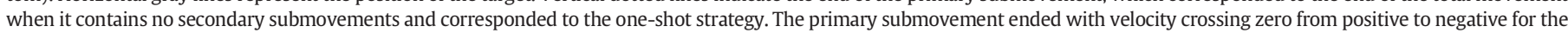

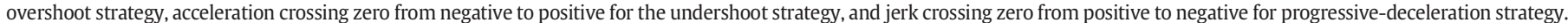


undershoot strategy (i.e., the participant underestimated the distance and had to make a secondary corrective submovement (reacceleration) to reach the target); (c) the progressive-deceleration strategy was determined by zero-crossing of the jerk profile (i.e., the participant performs a movement that brings his arm near the target and slowly approaches the target, leading to a lengthening of the deceleration phase). (d) When the end of the primary submovement coincided with the movement end (i.e., no corrections were detected) it was considered as the one-shot strategy.

\section{Results}

All trials containing errors ${ }^{1}$ ( $0 \%$ for easy ID and $29 \%$ for hard ID) were removed (analyses of mean percent errors revealed only a main effect of ID, $\mathrm{F}(1,46)=272.56, \mathrm{p}<.001)$. Moreover, the first trial of each block (6\%) was excluded. Also, trials where the overshoot strategy was used (since it was rarely used) were excluded from the ANOVA analyses (3\%). All reported effects are significant with $\mathrm{p}<.05$.

\subsection{Participants' performance and strategies}

To check whether our manipulation to test sequential effects did not change participants' approach to the task, we first analyzed participants' performance and strategies. We determined whether both young and older adults performed better on easier than on harder trials, whether older adults were slower and used fewer strategies than young adults, whether strategy distributions differed in each age group, and whether strategies yielded different levels of performance in both young and older adults.

\subsection{Age-related differences in movement times (MTs)}

An ANOVA was performed on movement times with a mixed design 2 (Group: young, older) $\times 2$ (Trial difficulty: easier, harder trials), with repeated measures on the last factor. MTs were significantly longer in older adults than in young adults $(705 \mathrm{~ms}$ vs. $501 \mathrm{~ms} ; \mathrm{F}(1,46)=$ $36.97, \mathrm{MSe}=26,990, \mathrm{n}^{2} \mathrm{p}=0.46$ ) and were longer for the harder trials compared to the easier trials (701 ms vs. $504 \mathrm{~ms} ; \mathrm{F}(1,46)=367.60$, $\left.\mathrm{MSe}=2537, \mathrm{n}^{2} \mathrm{p}=0.89\right)$. The Group $\times$ Trial interaction was not significant $(\mathrm{F}<1)$.

\subsubsection{Age-related differences in strategy repertoire}

An ANOVA with mixed designs, 2 (Group: young, older) $\times 2$ (Trial difficulty: easier, harder trials), and repeated measures on the last factor, was performed on the mean number of strategies used by individuals. Participants used significantly more strategies for harder trials than for easier trials ( 3.2 vs. 1.9 strategies; $F(1,46)=78.31$, MSe $=$ $\left.0.45, \mathrm{n}^{2} \mathrm{p}=0.63\right)$. The Group $\times$ Trial Difficulty interaction was significant $\left(\mathrm{F}(1,46)=20.95\right.$, MSe $\left.=0.45, \mathrm{n}^{2} \mathrm{p}=0.32\right)$, showing that older adults used more strategies than young adults for easier trials ( $2.3 \mathrm{vs.}$ 1.6 strategies; $F(1,46)=18.72$, MSe $=0.32, n^{2} p=0.29$ ), and fewer strategies for harder trials (2.9 vs. 3.4 strategies; $F(1,46)=7.21$, MSe $=0.49, \mathrm{n}^{2} \mathrm{p}=0.14$ ).

\subsubsection{Age-related differences in strategy distributions}

A three-way ANOVA, 2 (Group: young, older) $\times 2$ (Trial difficulty: easier, harder trials) $\times 3$ (Strategy: one-shot, undershoot, progressivedeceleration strategies), with repeated measures on the last two factors, was carried out on mean percentages of use of the three most often used strategies (i.e., the overshoot strategy, used on only $3 \%$ of trials, was

\footnotetext{
${ }^{1}$ As there are quite a few errors (14.8\%), we also investigated the impact of an error on the subsequent trial. We compared the percentage of use of each strategy after an erroneous trial vs. after a successful trial, in young and older adults, for both ID conditions. However, the statistical comparisons of the percentages of the different strategies in both situations failed to reveal any differences.
}

excluded). Results revealed a significant Group $\times$ Strategy interaction $\left(\mathrm{F}(2,92)=11.99, \mathrm{MSe}=555.0 ; \mathrm{n}^{2} \mathrm{p}=0.21\right)$. Older adults used more frequently than young adults the two strategies involving submovements (i.e., undershoot strategy - $25 \%$ vs. $16 \%$, and progressivedeceleration strategy - $33 \%$ vs. $22 \%$, in older and young adults, respectively), and less frequently the one-shot strategy (39\% vs. $58 \%$; all Fs $>5.23$ comparing young and older adults on each strategy). Also, the Group $\times$ Trial Difficulty $\times$ Strategy interaction came out significant $\left(\mathrm{F}(2,92)=4.02, \mathrm{MSe}=236.4 ; \mathrm{n}^{2} \mathrm{p}=0.08\right)$. This interaction resulted from similar strategy preferences in young and older adults for the easier trials but not for the harder trials (see Fig. 3).

On easier trials, young adults and older adults used the one-shot strategy the most often ( $88 \%$ and $69 \%$, respectively) and the undershoot strategy the least often ( $0 \%$ and $3 \%$ ), with the progressive-deceleration strategy in-between ( $11 \%$ and $29 \%$ ). Conversely, on harder trials, young adults used the three strategies equally often (28\%, 33\%, and 32 , for the one-shot, the undershoot, and the progressive-deceleration strategies, respectively). Older adults used the undershoot strategy the most often (48\%) and the one-shot strategy the least often (10\%), with the progressive-deceleration strategy in-between (37\%). On easier trials, compared to young adults, older adults used the undershoot and progressive-deceleration strategies more often $(\mathrm{Fs}>8.56)$ than the one-shot strategy $(F(1,46)=9.46)$. On harder trials, older adults used the undershoot strategy more often than young adults $(F(1,46)=$ $15.42)$, the progressive deceleration strategy equally often $(\mathrm{F}<1)$, and the one-shot strategy less often $(F(1,46)=20.94)$.

\subsubsection{Age-related differences in strategy execution}

A two-way ANOVA (Group $\times$ Strategy), with repeated-measures on the last factor was carried out on mean movement times. As shown in Fig. 4, MTs were shorter in young adults than older adults (551 ms vs. $693 \mathrm{~ms} ; \mathrm{F}(1,46)=24.23$, MSe $\left.=29,621, \mathrm{n}^{2} \mathrm{p}=0.36\right)$. A main effect of strategy was found $\left(\mathrm{F}(2,92)=402.58, \mathrm{MSe}=1681, \mathrm{n}^{2} \mathrm{p}=0.90\right)$. Pairwise comparisons revealed that MTs were shorter with the oneshot strategy than with the progressive-deceleration strategy $(\mathrm{F}(1,46)=77.30)$, or than with the undershoot strategy $(\mathrm{F}(1,46)=$ $625.41)$. Also, MTs were shorter with the progressive-deceleration strategy than with the undershoot strategy $(F(1,46)=354.43)$. The Group $\times$ Strategy interaction was significant $(\mathrm{F}(2,92)=4.61$, MSe $=$ $1681, \mathrm{n}^{2} \mathrm{p}=0.09$ ), showing that young adults were faster than older adults whatever the strategies they used (Fs > 13.36). However, age differences varied with strategy such that they were largest for the oneshot strategy $\left(F(1,46)=28.05\right.$, MSe $\left.=11,506.8, n^{2} \mathrm{p}=0.38\right)$ and lowest for the undershoot strategy $(\mathrm{F}(1,46)=13.36$, MSe $=11,631.8$, $\mathrm{n}^{2} \mathrm{p}=0.23$ ), with the progressive-deceleration strategy in-between $\left(\mathrm{F}(1,46)=25.90, \mathrm{MSe}=9844.2, \mathrm{n}^{2} \mathrm{p}=0.36\right)$.

In sum, the present results showed that older adults took more time than young adults to perform the Fitts' task. Moreover, both age groups used the same repertoire of four strategies. However, compared to young adults, older adults used fewer strategies for easier trials and

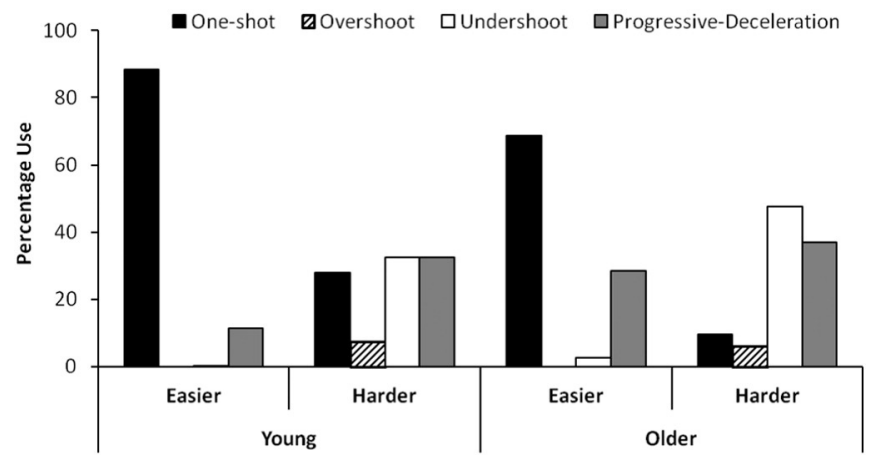

Fig. 3. Mean percentages of use of each strategy in young and older adults for the easier and harder trials. 


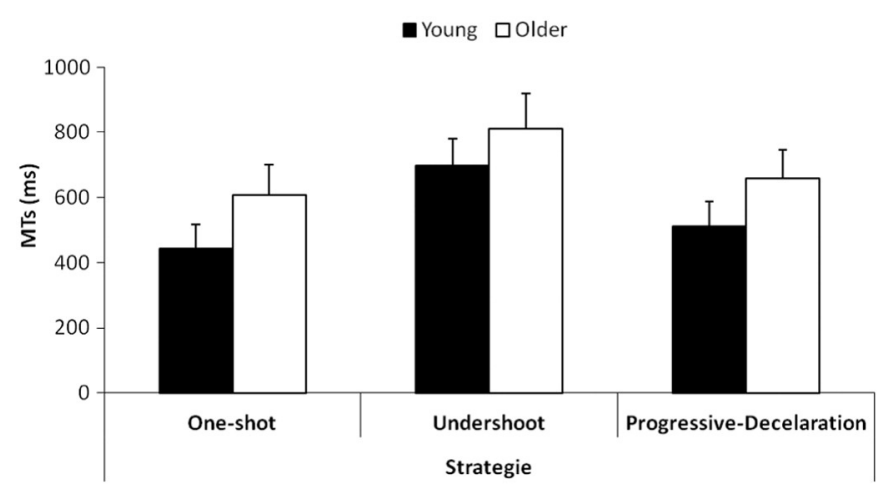

Fig. 4. Mean movement times (MTs) in ms, in young and older adults while using the oneshot, undershoot, or progressive-deceleration strategies.

more strategies for harder trials. Also, we found that strategy use varied across trial difficulty and participants' age. Participants of both age groups predominantly used the one-shot strategy on easier trials, whereas on harder trials, young adults used the three strategies equally and older adults preferred strategy including corrective submovements (i.e., the undershoot and progressive-deceleration strategies). Finally, we found that the different strategies that participants used differed in relative efficacy. Moreover, although the hierarchy of relative strategy difficulty was the same in young and older adults, age-related differences were modulated by trial difficulty and tended to be larger for the easier strategy (i.e., the one-shot strategy). Thus, despite the difference in the experimental design, these findings are in line with those observed in our previous study (Poletti et al., 2015) with regard to performance, strategy repertoire, and strategy execution. However, different strategy distributions were found compared to our previous study. Indeed young adults used the one-shot strategy most often and older adults preferred the progressive-deceleration strategy to perform easier trials, whereas on harder trials, both age groups predominantly used the progressive-deceleration strategy.

\subsection{Age-related differences in sequential effects}

\subsubsection{Age-related differences in trial sequential difficulty effects (TSDE)}

ANOVAs were performed on mean movement times ${ }^{2}$ on current trials with mixed designs, 2 (Group: young, older adults) $\times 2$ (Previous difficulty trial: easier, harder trials), with repeated measures on the last factor. As it can been seen in Fig. 5, MTs were longer in older adults than in young adults (688 ms vs. $493 \mathrm{~ms} ; \mathrm{F}(1,46)=36.69$, MSe $=$ $29,157, n^{2} p=0.43$ ). Moreover, MTs were longer on current trials after harder trials than after easier trials (598 ms vs. $573 \mathrm{~ms}$; $\mathrm{F}(1,46)=49.07$, MSe $=303, \mathrm{n}^{2} \mathrm{p}=0.52$ ). The interaction between age and previous difficulty trials was not significant $(\mathrm{F}<1)$, revealing significant TSDE in each age group and TSDE of comparable magnitudes in young $(+23 \mathrm{~ms})$ and older $(+27 \mathrm{~ms})$ adults.

\subsubsection{Age-related differences in strategy sequential difficulty effects (SSDE)}

We conducted a two-way ANOVA on participants' mean movement times $^{3}$ on current trials when they used the strategy of intermediate difficulty (i.e., progressive-deceleration strategy) following trials on which they used the easier, one-shot strategy versus trials on which they used the harder, undershoot strategy. The mixed-design ANOVA was carried out with the strategy used on previous trials as the only withinparticipants factor. MTs were longer in older adults than young adults

\footnotetext{
2 The same analyses were performed on proportions TSDE, (i.e., [MTs after hard trial - MTs after easy trial] / MTs after easy trial $\times 100$ ), and the same results were found. 3 The same analyses were performed on proportion of SSDE, (i.e., [MTs after undershoot strategy - MTs after one-shot strategy] / MTs after one-shot strategy $\times 100)$, and the same results were found.
}

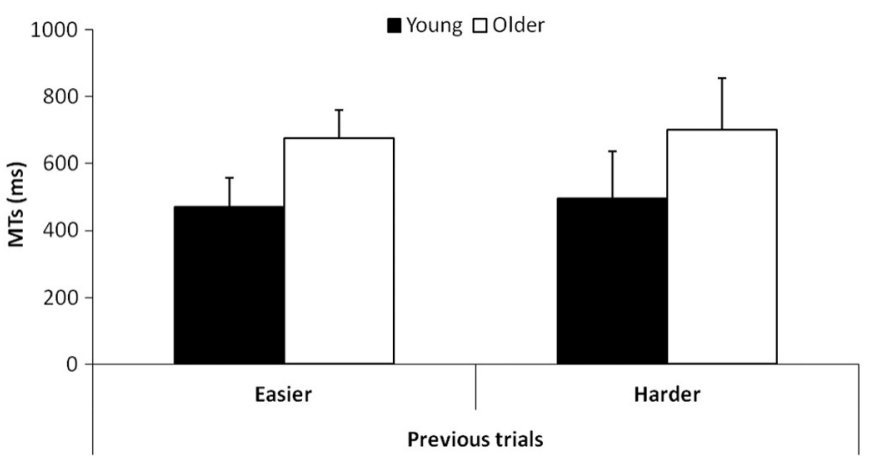

Fig. 5. Mean movement times (in ms) on current trial as a function of the difficulty of previous trials, in young and older adults.

( 660 ms vs. 536 ms; $F(1,46)=22.02$, MSe $=16,913, \mathrm{n}^{2} \mathrm{p}=0.32$ ). Moreover, MTs were longer with the progressive-deceleration strategy when this strategy followed the undershoot strategy than after the one-shot strategy (616 ms vs. $581 \mathrm{~ms} ; \mathrm{F}(1,46)=14.46, \mathrm{MSe}=2015, \mathrm{n}^{2} \mathrm{p}=$ $0.24)$. The interaction between age and previous strategy was not significant, $\mathrm{F}(1,46)=2.3$ (see Table 2). Although in absolute terms strategy sequential difficulty effects tended to be larger in older than in young adults (+48 ms vs. $+21 \mathrm{~ms}$ ), the two age groups showed nosignificantly different strategy sequential difficulty effects.

\subsubsection{Age-related differences in strategy repetition effects (SRE)}

Given strategy selection (e.g., participants never repeated the undershoot strategy on easier trials), we collapsed over easier and harder trials to analyze mean percentages of strategy repetitions (i.e., if participants used the same strategy on current and previous trials, strategy repetition was coded 1 ; otherwise, it was coded 0 ). An ANOVA was performed on the mean percentages of strategy repetitions with a mixed design: 2 (Group: young, older adults) $\times 3$ (Strategy: one-shot, undershoot, and progressive-deceleration strategies), with repeated measures on the last factor. Overall, a main effect of strategy was found $\left(\mathrm{F}(2,92)=52.74\right.$, MSe $\left.=737.7, \mathrm{n}^{2} \mathrm{p}=0.53\right)$. Pairwise comparisons revealed that participants tended to repeat the one-shot strategy the most often $(F(1,46)=139.96)$ and the undershoot strategy the least often $(F(1,46)=29.0)$, with the progressive-deceleration strategy in-between $(F(1,46)=14.73)$. The Group $\times$ Strategy interaction was significant $\left(\mathrm{F}(2,92)=9.87, \mathrm{MSe}=737.7, \mathrm{n}^{2} \mathrm{p}=0.17\right)$, showing that older adults tended to repeat most often the undershoot $(\mathrm{F}(1,46)=$ $14.46)$ and progressive-deceleration strategies $(F(1,46)=6.17)$ and less often the one-shot strategy $(F(1,46)=12.56)$ than young adults (see Fig. 6).

In sum, these findings showed the existence of trial-based and strategy-based sequential difficulty effects in both young and older adults. MTs were longer on current trials after performing harder trials or harder strategy relative to easier trials or easier strategy. Also, we found that these sequential difficulty effects were similar in both young and older adults. Moreover, age-related differences in strategy repetition effects varied according to the strategy (e.g., SRE were larger with the one-shot strategy).

\section{Table 2}

Mean movement times (MTs) in ms on current trials when participants used the progressive-deceleration strategy as a function of the strategy executed on previous trials, in young and older adults.

\begin{tabular}{lcc}
\hline Strategy used on previous trial & Young adults & Older adults \\
\hline One-shot (OS) & 525 & 636 \\
Undershoot (US) & 546 & 684 \\
SSDE (US-OS) & 21 & 48 \\
\hline
\end{tabular}




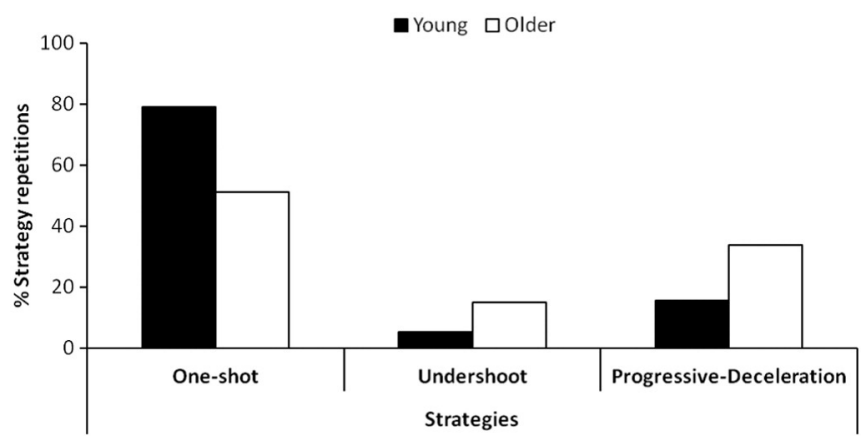

Fig. 6. Mean percentages of strategy repetitions in young and older adults.

\section{Discussion}

To contribute to our understanding of sensori-motor performance and aging effects thereon, the present study further investigated strategic variations in Fitts' task and, most originally, documented sequential effects in young and older adults.

The first step was to replicate our previous findings regarding strategic variations in Fitts' task (Poletti et al., 2015), despite some changes in our design undertaken to examine sequential effects. Indeed, our previous findings demonstrated significant age-related differences in strategy repertoire (i.e., older adults used fewer strategies than young adults), strategy distributions (i.e., older adults predominantly used strategies involving corrective submovements, whereas young adults preferred a strategy without corrective submovements), and strategy execution (i.e., older adults were slower than young adults whatever the strategies they used). Here, we found similar results to those obtained in our previous experiment regarding performance, strategy repertoire, strategy distributions, and strategy execution, as well as aging effects therein. Thus, taken together, these two studies provide evidence supporting the robustness of strategic variations in Fitts' aiming task. These results allowed us to be more confident with the investigation of sequential effects, which is one aspect of strategic variations. Three types of sequential effects were observed in both young and older adults: Trial sequential difficulty effects (TSDE) and strategy sequential difficulty effects (SSDE) occurred during strategy execution; and strategy repetition effects (SRE) were found during strategy selection. We next discuss these effects, their interpretation, and their implications on understanding of sensori-motor performance as well as age-related differences in this performance.

\subsection{Sequential effects in Fitts' task}

Performance on a given trial was influenced by the difficulty of the preceding trial (trial sequential difficulty effects; TSDE) and by the difficulty of the strategy used on the preceding trial (strategy sequential difficulty effects; SSDE). Specifically, our results showed that MTs were longer on current trials after performing harder trials or a harder strategy (i.e., the undershoot strategy) compared to easier trials or an easier strategy (i.e., the one-shot strategy). Moreover, we found that participants tended to repeat the same strategy across two consecutive trials (strategy repetition effects; SRE). These SRE varied with the target strategy, as participants repeated the one-shot strategy the most often (65\%) and the undershoot strategy the least often (10\%), with the progressivedeceleration in-between (25\%).

To our knowledge, sequential effects in strategic variations were never investigated in the motor control literature. Thus, the present findings extend to the sensori-motor domain, sequential effects previously observed in the cognitive domain (i.e., arithmetic problem solving and episodic memory) by Schneider and Anderson (2010); Uittenhove and Lemaire (2012), and Uittenhove, Burger, Taconnat, and Lemaire (2014).
The sequential effects in Fitts' aiming task could be explained by a common mechanism to cognitive and sensori-motor domains, although the functional resources involved in the related tasks are presumably different. Indeed, a plausible interpretation lies in the resource depletion hypothesis that has been suggested by Schneider and Anderson (2010) and further elaborated by Uittenhove and Lemaire (2012); Uittenhove et al., 2014) to explain sequential effects observed in the cognitive domain. According to the resource depletion hypothesis, sequential modulations of performance reflects the amount of resources still available to perform the task on a current trial following an easy or a difficult task/strategy on the immediately preceding trial. Specifically, with limited amount of resources, participants have used up most of their available resources to perform a difficult trial/strategy on a given trial. Subsequently, the available amount of resources on the next trial is depleted, thereby decreasing their performance in this next trial. Another result observed in the present study during strategy choices is consistent with the resource depletion hypothesis. Indeed, participants more frequently repeated the one-shot strategy, whereas they switched strategy after using either undershoot or progressive-deceleration strategies. This could be explained by the fact that using undershoot or progressive-deceleration strategies on a given trial was more resource-consuming than using the one-shot strategy. As a consequence, fewer resources remained available after using a difficult strategy, which might have led participants to switch to a strategy that consumed fewer resources. In sum, our results showed that both strategy execution and strategy choices are influenced by trial or strategy transitions (i.e., the nature of successive trials and strategies).

The question that remains however concerns the nature of the resources that might have been depleted during sequential repetitions of aiming movements. A plausible hypothesis lies in the temporary reduction in executive control capacities in addition to multisensory integration functions, which are known to be involved during the deceleration phase of aiming movements (see Elliott et al., 2001, for a review). Indeed, recent studies suggested that executive functions are prominently involved in complex motor tasks (e.g., locomotion; Verghese, Mahoney, Ambrose, Wang, \& Holtzer, 2010; Yogev-Seligmann, Hausdorff, \& Giladi, 2008; posture; Woollacott \& Shumway-Cook, 2002) and, in particular, in Fitts' aiming task (Rey-Robert et al., 2012; Sleimen-Malkoun et al., 2013; Temprado et al., 2013). Thus, under conditions taxing executive control resources, task-related executive processes could be more depleted after performing a harder trial/strategy than after an easier trial/strategy. Although we did not collect any measures of executive functions in the present study, this interpretation remains to be directly tested in future studies. Note that it is consistent with findings observed in many cognitive domains, which showed that SSDE were related to the efficiency of executive functions in both young and older adults (Uittenhove et al., 2014).

\subsection{Age-related differences in sequential effects}

In contrast to lack of age differences in sequential effects during strategy execution, the present data showed age-related differences in sequential effects during strategy choices. As Lemaire and Leclère (2014a, 2014b), who used an arithmetic problem solving task where strategy choices are controlled, we found SRE that is, a tendency to use the same strategy over two successive trials, in both young and older adults. Moreover, SRE varied with the type of strategy, and differently in young and older adults. Indeed, SRE were larger with undershoot or progressive-deceleration strategies in older adults as compared to young adults. Conversely, SRE were smaller with the one-shot strategy in older than in young adults. In other words, on the basis of these results, we could conclude that older adults tended to repeat the harder strategies (i.e., the undershoot and progressivedeceleration strategies) more often than young adults, but the easier strategy (i.e., the one-shot strategy) less often. To explain these results, it can be suggested that because the one-shot strategy is easier, older 
adults had fewer difficulties to switch strategy from one trial to the next, after using it. In contrast, after using the harder undershoot or progressive-deceleration strategies on a given trial, they had fewer resources left free to switch strategy on the next trial, leading them to repeat these strategies.

However, it is noticeable that there are unequal proportions of strategies used by participants. Thus, SRE could be a consequence of the difference in the proportions of strategies used by young and older participants. Indeed, the one-shot strategy was used more by younger than by older participants and vice versa for the other two strategies. As a consequence, it could appear logical that one-shot strategy is repeated more often by young than by older participants, and vice versa for the other two strategies. Future studies could control age-related differences in strategy distribution. This was not the case in the present study since, in Fitts' task, the control of the strategies (e.g., via instructions) that participants execute is impossible. Note though that in other cognitive tasks, like arithmetic problem solving task, when agerelated differences in strategy distributions were controlled, older adults tended to repeat the same strategies across consecutive trials more often than young adults (e.g., Lemaire \& Leclère, 2014a, 2014b).

In sum, the present findings originally showed an age-dissociation in sequential effects between strategy execution and strategy selection. Difference between age-related modulations of sequential effects during strategy selection on the one hand, and lack of age differences in sequential effects during strategy execution on the other hand, could be most likely due to extra-processing demands induced by strategy selection. Consistent with previous findings (e.g., Bouazzaoui et al., 2010; Hodzik \& Lemaire, 2011; Taconnat et al., 2006) showing that agerelated differences in executive functions mediate age-related differences in best strategy selections on each trial, it is possible that the well-known age-related decrease in executive control processes (see Jurado \& Rosselli, 2007, for a review), combined with greater processing demands during strategy selection, led to increased age-related differences in sequential effects during strategy selection relative to during strategy execution.

\subsection{Conclusion and perspectives}

The present study successfully extended our previous findings (Poletti et al., 2015) to an experiment specifically designed for the original investigations of sequential effects. Thus, strategic variations in the Fitts' task seem to be robust.

Three different sequential effects were observed, two concerning strategy execution (i.e., trial sequential difficulty effects showing that MTs were longer after performing a difficult trial; and strategy sequential difficulty effects showing that MTs were longer after performing a difficult strategy); and one during strategy selection (i.e., strategy repetition effects revealing that participants tended to repeat the same strategy across two consecutive trials). These sequential effects could be explained by a general mechanism of resource depletion from trial to trial. Moreover, we found an age-dissociation on these sequential effects. Age-invariance in sequential effects during strategy execution occurred together with larger sequential effects in older adults during strategy selection.

Overall, these findings have significant implications for the understanding of the processes underlying sensori-motor performance in young and older adults. First, they demonstrate, for the first time to our knowledge, that sequential effects are not restricted to cognitive tasks; they can be generalized to the sensori-motor domain. Second, these findings raise the question of whether sequential effects can be observed in other sensori-motor tasks, and if affirmative, whether they can explain the results observed previously in the literature. Finally, future studies could decipher the exact mechanisms for how and why performing difficult trials/strategies in quick successions can negatively affect participants' performance.

\section{References}

Bashore, T., Ridderinkhof, K., \& van der Molen, M. (1997). The decline of cognitive processing speed in old age. Current Directions in Psychological Science, 6, 166-169.

Birren, J.E., Woods, A.M., \& Williams, M.V. (1980). Behavioral slowing with age: Causes, organization, and consequences. In L.W. Poon (Ed.), Aging in the 1980s (pp. 293-308). Washington, DC: American Psychological Association.

Bouazzaoui, B., Isingrini, M., Fay, S., Angel, L., Vanneste, S., Clarys, D., \& Taconnat, L. (2010) Aging and self-reported internal and external memory strategy uses: The role of executive functioning. Acta Psychologica, 135(1), 59-66.

Cerella, J. (1985). Information processing rates in the elderly. Psychological Bulletin, 98(1), 67-83.

Dounskaia, N., Wisleder, D., \& Johnson, T. (2005). Influence of biomechanical factors on substructure of pointing movements. Experimental Brain Research, 164(4), 505-516.

Dunlosky, J., Hertzog, C., \& Powell-Moman, A. (2005). The contribution of mediator-based deficiencies to age differences in associative learning. Developmental Psychology, 41(2), 389-400.

Elliott, D., Hansen, S., Grierson, L.E.M., Lyons, J., Bennett, S.J., \& Hayes, S.J. (2010). Goaldirected aiming: Two components but multiple processes. Psychological Bulletin. 136(6), 1023-1044.

Elliott, D., Helsen, W.F., \& Chua, R. (2001). A century later: Woodworth's (1899) twocomponent model of goal-directed aiming. Psychological Bulletin, 127(3), 342-357.

Fitts, P. M. (1954). The information capacity of the human motor system in controlling the amplitude of movement. Journal of Experimental Psychology, 47, 381-391.

Fitts, P. M., \& Peterson, J. R. (1964). Information capacity of discrete motor responses. Journal of Experimental Psychology, 67, 103-112.

Fradet, L., Lee, G., \& Dounskaia, N. (2008). Origins of submovements in movements of elderly adults. Journal of Neuroengineering and Rehabilitation, 5, 28.

Folstein, M.F., Folstein, S.E., \& McHugh, P.R. (1975). "Mini-mental state": A practical method for grading the cognitive state of patients for the clinician. Journal of Psychiatric Research, 12, 189-198.

Gandini, D., Lemaire, P., \& Dufau, S. (2008). Older and younger adults' strategies in approximate quantification. Acta Psychologica, 129(1), 175-189.

Haaland, K.Y., Harrington, D.L., \& Grice, J.W. (1993). Effects of aging on planning and implementing arm movements. Psychology and Aging, 8(4), 617-632.

Hodzik, S., \& Lemaire, P. (2011). Inhibition and shifting capacities mediate adults' agerelated differences in strategy selection and repertoire. Acta Psychologica, 137(3), 335-344.

Jurado, M.B., \& Rosselli, M. (2007). The elusive nature of executive functions: A review of our current understanding. Neuropsychology Review, 17, 213-233.

Ketcham, C.J., Seidler, R.D., Van Gemmert, A.W.A., \& Stelmach, G.E. (2002). Age-related kinematic differences as influenced by task difficulty, target size, and movement amplitude. Journal of Gerontology: Psychological Sciences, 57B(1), 54-64.

Lemaire, P. (2010). Cognitive strategy variations during aging. Current Directions in Psychological Science, 19(6), 363-369.

Lemaire, P. 2015. Cognitive Aging: The Role of Strategies. London: Psychology Press (in press).

Lemaire, P., \& Arnaud, L. (2008). Young and older adults' strategies in complex arithmetic. American Journal of Psychology, 121, 1-16.

Lemaire, P., Arnaud, L., \& Lecacheur, M. (2004). Adults' age-related differences in adaptivity of strategy choices: Evidence from computational estimation. Psychology and Aging, 19(3), 467-481.

Lemaire, P., \& Leclère, M. (2014a). Strategy repetition in young and older adults: A study in arithmetic. Developmental Psychology, 50(2), 460-468.

Lemaire, P., \& Leclère, M. (2014b). Strategy selection in Alzheimer patients: A study in arithmetic. Journal of Experimental and Clinical Neuropsychology, 36(5), 507-516.

Lemaire, P., \& Reder, L. (1999). What affects strategy selection in arithmetic? The example of parity and five effects on product verification. Memory $\mathcal{E}$ Cognition, 27(2), 364-382.

Lemaire, P., \& Siegler, R. S. (1995). Four aspects of strategic change: Contributions to children's learning of multiplication. Journal of Experimental Psychology: General, 124(1), 83-97.

MacKenzie, S.I. (1992). Fitts' law as a research and design tool in human-computer interaction. Human Computer Interaction, 7, 91-139.

Meyer, D.E., Abrams, R.A., Kornblum, S., Wright, C.E., \& Smith, J.E.K. (1988). Optimality in human motor performance: Ideal control of rapid aimed movements. Psychological Review, 95(3), 340-370.

Poletti, C., Sleimen-malkoun, R., Temprado, J. -J., \& Lemaire, P. (2015). Older and younger adults' strategies in sensorimotor tasks: Insights from fitts' pointing task. Journal of Experimental Psychology: Human Perception and Performance, 41(2), 542-555.

Pratt, J., Chasteen, A.L., \& Abrams, R.A. (1994). Rapid aimed limb movements: Age differences and practice effects in component submovements. Psychology and Aging, 9(2), 325-334.

Rey-Robert, B., Temprado, J. -J., Lemaire, P., \& Berton, E. (2012). Combining movement kinematics, efficiency functions, and Brinley plots to study age-related slowing of sensorimotor processes: Insights from Fitts' task. Gerontology, 58(2), $171-180$.

Salthouse (1996). The processing-speed theory of adult age differences in cognition. Psychological Review, 103(3), 403-428.

Schneider, D.W., \& Anderson, J.R. (2010). Asymmetric switch costs as sequential difficulty effects. Quarterly Journal of Experimental Psychology, 63(10), 1873-1894.

Sleimen-Malkoun, R., Temprado, J.J., Huys, R., Jirsa, V., \& Berton, E. (2012). Is Fitts' law continuous in discrete aiming? PloS One, 7(7), e41190.

Sleimen-Malkoun, R., Temprado, J. -J., \& Berton, E. (2013). Age-related changes of movement patterns in discrete Fitts' task. BMC Neuroscience, 14, 145. 
Taconnat, L., Baudouin, A., Fay, S., Clarys, D., Vanneste, S., Tournelle, L., \& Isingrini, M. (2006). Aging and implementation of encoding strategies in the generation of rhymes: The role of executive functions. Neuropsychology, 20(6), 658-665.

Teasdale, N., Bard, C., Fleury, M., Young, D.E., \& Proteau, L. (1993). Determining movement onsets from temporal series. Journal of Motor Behavior, 25(2), 97-106.

Teeken, J.C., Adam, J.J., Paas, F.G.W.C., Boxtel, M.P.J.V., Houx, P.J., \& Jolles, J. (1996). Effects of age and gender on discrete and reciprocal aiming movements. Psychology and Aging, 11(2), 195-198.

Temprado, J. -J., Sleimen-Malkoun, R., Lemaire, P., Rey-Robert, B., Retornaz, F., \& Berton, E (2013). Aging of sensorimotor processes: A systematic study in Fitts' task. Experimental Brain Research, 228(1), 105-116.

Uittenhove, K., Burger, L., Taconnat, L., \& Lemaire, P. (2014). Sequential difficulty effects during execution of memory strategies in young and older adults. Memory, 23(6), 806-816.

Uittenhove, K., \& Lemaire, P. (2012). Sequential difficulty effects during strategy execution. Experimental Psychology, 59(5), 295-301.

Uittenhove, K., \& Lemaire, P. (2013a). Strategy sequential difficulty effects in Alzheimer patients: A study in arithmetic. Journal of Clinical and Experimental Neuropsychology, $35,83-89$.

Uittenhove, K., \& Lemaire, P. (2013b). Strategy sequential difficulty effects vary with working-memory and response-stimulus-intervals: A study in arithmetic. Acta Psychologica, 143, 113-118.
Uittenhove, K., Poletti, C., Dufau, S., \& Lemaire, P. (2013). The time course of strategy sequential difficulty effects: An ERP study in arithmetic. Experimental Brain Research, 227(1), 1-8.

Van Halewyck, F., Lavrysen, A., Levin, O., Boisgontier, M.P., Elliott, D., \& Helsen, W.F. (2014). Both age and physical activity level impact on eye-hand coordination. Human Movement Science, 36, 80-96.

Van Halewyck, F., Lavrysen, A., Levin, O., Boisgontier, M.P., Elliott, D., \& Helsen, W.F. (2015). Factors underlying age-related changes in discrete aiming. Experimental Brain Research, 233(6), 1733-1744.

Verghese, J., Mahoney, J., Ambrose, A.F., Wang, C., \& Holtzer, R. (2010). Effect of cognitive remediation on gait in sedentary seniors. Journals of Gerontology. Series A, Biological Sciences and Medical Sciences, 65, 1338-1343.

Woodworth, R. S. (1899). Accuracy of voluntary movement. The Psychological Review: Monograph Supplements, 3(3), 1-114.

Woollacott, M., \& Shumway-Cook, A. (2002). Attention and the control of posture and gait: A review of an emerging area of research. Gait \& Posture, 16, 1-14.

Welford, A.T., Norris, A.T., \& Shock, N.W. (1969). Speed and accuracy of movement and their changes with age. Acta Psychologica, 30, 3-15.

Yogev-Seligmann, G., Hausdorff, J.M., \& Giladi, N. (2008). The role of executive function and attention in gait. Movement Disorders, 23(3), 329-342.

York, J., \& Biederman, I. (1990). Effects of age and sex on reciprocal tapping performance. Perceptual and Motor Skills, 71, 675-684. 